\title{
The Impact of Physician EHR Usage on Patient Satisfaction
}

\author{
Rebecca A. Marmor ${ }^{1}$ Brian Clay ${ }^{2}$ Marlene Millen ${ }^{2}$ \\ ${ }^{1}$ Department of Biomedical Informatics and Surgery, University of \\ California San Diego, San Diego, California, United States \\ ${ }^{2}$ Department of Biomedical Informatics and Medicine, University of \\ California San Diego, San Diego, California, United States \\ ${ }^{3}$ Department of Medicine, University of California San Diego, \\ San Diego, California, United States \\ ${ }^{4}$ Department of Biomedical Informatics and Pediatrics, University of \\ California San Diego, San Diego, California, United States
}

Appl Clin Inform 2018;9:11-14.

\section{Background and Significance}

The increased emphasis on patient satisfaction has coincided with the growing adoption of electronic health records (EHRs) throughout the U.S. The 2001 Institute of Medicine Report, "Crossing the Quality Chasm," identified patient-centered care as a key element of quality health care. ${ }^{1}$ In response to this call, the Hospital Consumer Assessment of Healthcare Providers and Systems (HCAHPS) survey was developed to assess patients' health care experiences in the inpatient setting. Simultaneously, financial incentives have facilitated the rapid adoption of EHR applications, with $84 \%$ of hospitals maintaining at least a basic EHR in 2015 (a ninefold increase since 2008). ${ }^{2}$

Despite the concurrent deployment of patient satisfaction surveys and EHRs, there is a poor understanding of the relationship that may exist between physician usage of the EHR and patient satisfaction. Most prior research into the impact of the EHR on physician-patient communication has been observational, describing the behaviors of physicians and patients when the clinician accesses an EHR in the exam room. Past research has shown that encounters where physicians access the EHR are often filled with long pauses, ${ }^{3}$ and that few clinicians attempt to engage patients by sharing what they are looking at on the screen. ${ }^{4} \mathrm{~A}$ recent metaanalysis reviewing 53 papers found that only 7 studies attempted to correlate objective observations of physician communication behaviors with patient perceptions by eliciting feedback from the patients. ${ }^{5}$ No study used a standardized assessment tool of patient satisfaction. The authors conclude that additional work is necessary to better understand the patient perspective of the presence of an EHR during a clinical encounter.

received

June 16, 2017

accepted after revision

November 15, 2017

\author{
Thomas J. Savides ${ }^{3}$ Christopher A. Longhurst ${ }^{4}$
}

Address for correspondence Rebecca A. Marmor, MD, MAS, Department of Biomedical Informatics and Surgery, University of California San Diego, 200 W. Arbor Dr., San Diego, CA 92103, United States (e-mail: Rmarmor@ucsd.edu).

Additionally, increasing EHR adoption and emphasis on patient satisfaction have also corresponded with rising physician burnout rates. ${ }^{6,7}$ Prior work suggests that EHR adoption may be contributing to this trend. ${ }^{8}$ Burnout from the EHR may be due in part to the significant amount of time physicians spend logged into systems, documenting long after clinic has ended in effort to avoid disrupting the patient-physician relationship. ${ }^{9}$

We used existing data sources to describe the relationship between the amount of time physicians spend logged in to the EHR-both during daytime hours as well after clinic hours -and performance on a validated patient satisfaction survey. Our null hypothesis is that there is no relationship between increased time logged in to the EHR and patient satisfaction.

\section{Methods}

Our work was determined to be exempt by our Institutional Review Board for quality improvement purposes. We queried EHR system reports to obtain the average monthly hours attending physicians from general internal medicine $(n=27)$, cardiology $(n=10)$, and gastroenterology $(n=2)$ outpatient clinics at a single academic institution spent logged into the EHR both during and outside of normal clinic hours from March 2015 to March 2016. Additionally, we obtained the number of days per month the physicians had outpatient clinics. We also obtained provider performance reports from the Clinician and Group Consumer Assessment of Healthcare Providers and Systems (CG CAHPS) survey from the same period. The CG CAHPS survey is the ambulatory version of the HCAHPS survey, designed to assess patient satisfaction with outpatient clinic encounters. ${ }^{10}$ 
Providers were excluded from the analysis if they had fewer than 30 surveys returned during the study period. Information about survey participants including diagnoses, age, sex, etc. was unavailable to protect the anonymity of the participants.

Our primary outcome was the providers' score on the likelihood to recommend provider item. Likelihood to recommend provider is a common surrogate for overall satisfaction with care. ${ }^{11}$ Secondary outcomes include the providers' score on the communication domain of the CG CAHPS. The communication domain was chosen because the questions asked are most closely aligned to providers' usage of EHR. This domain was comprised of an overall communication score, as well as four subdomains: (1) provider knew important medical history, (2) provider spent enough time with the patient, (3) provider showed respect for what patient said, and (4) provider listened carefully to patient.

We used descriptive statistics to characterize both the providers' overall average monthly EHR usage during normal clinic and after-hours as well as providers' monthly average hours of EHR usage per day of clinic. We performed univariable regressions to assess the relationship between duration of EHR usage and performance on the CG CAHPS domains. We calculated an $R^{2}$ to assess for the strength of the relationships. Statistical analyses were performed in SPSS version 24.

\section{Results}

\section{Differences in EHR Usage between Specialties}

General internists and specialists spent an average of 3.4 (2.5) hours per clinic day logged into the EHR during daytime hours and 0.79 (0.78) hours per clinic day logged in to the EHR after normal clinic hours ( - Fig. 1). General internists spent the highest absolute number of after-hours per month in the EHR [25.5 (SD: 24.2)] followed by gastroenterologists [18.2 (SD: 24.5)].

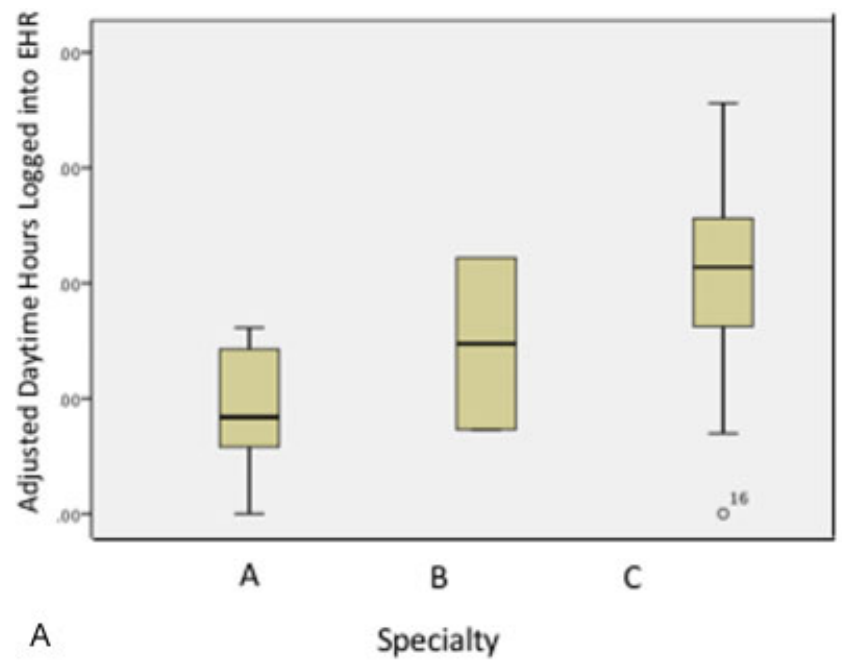

\section{After-Hours EHR Usage and Patient Satisfaction}

We found no association between duration of after-hours EHR usage and performance on the CG CAHPS. Increased after-hours usage was not associated with provider performance on the following CG CAHPS questions: (1) provider spent enough time with patient $\left(R^{2}=0.031\right)$, (2) provider showed patient respect $\left(R^{2}=0.000\right)$, (3) provider knew patient's history $\left(R^{2}=0.003\right),(4)$ provider listened carefully to patient $\left(R^{2}=0.006\right)$, (5) overall communication quality $\left(R^{2}=0.004\right)$, and (6) likelihood to recommend provider $\left(R^{2}=0.003\right)$. Univariable regression confirmed that afterhours usage had no association with survey performance.

\section{Daytime EHR Usage and Patient Satisfaction}

There appeared to be a small, but statistically significant, inverse relationship between daytime hours spent logged into the EHR and CG CAHPS scores for several domains including: (1) provider showed patient respect $\left(R^{2}=0.119, p=0.040\right)$, (2) provider knew patient's history $\left(R^{2}=0.174, p=0.011\right)$, (3) overall communication quality $\left(R^{2}=0.130, p=0.031\right)$, and (4) likelihood to recommend provider $\left(R^{2}=0.144, p=0.023\right)$ (-Fig. 2). There was no statistically significant association between daytime EHR usage and CG CAHPS outcomes for: (1) provider spent enough time with patient and (2) provider listened carefully to patient.

\section{Conclusion}

Our pilot study has found a small, but statistically significant, inverse relationship between daytime EHR usage and patient satisfaction scores for general internists and medicine sub specialists. This effect was observed for several components of the CG CAHPS communication domain as well as for likelihood to recommend the provider. Additionally, we did not identify an association between increased after-hours EHR usage and patient satisfaction.

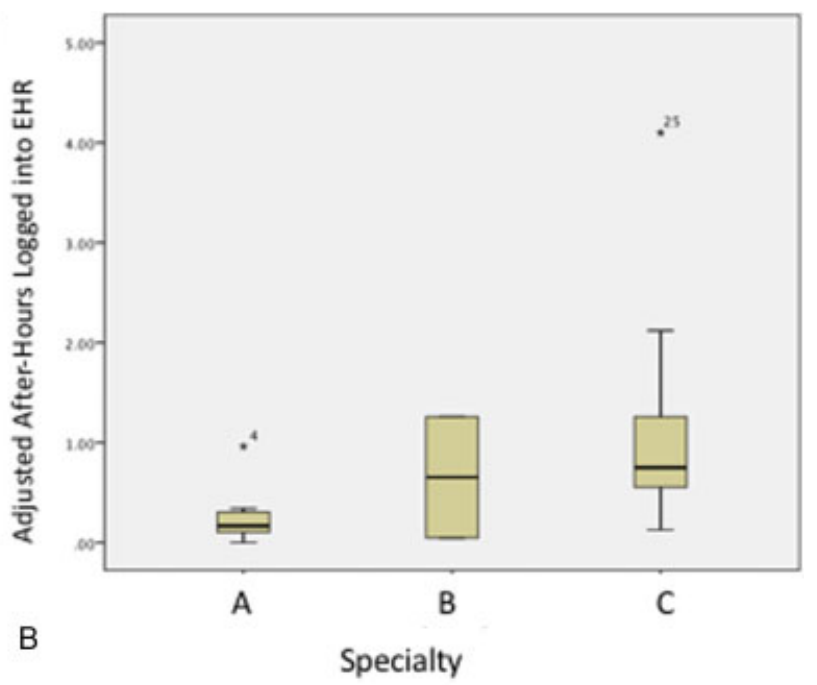

A- Cardiology B- Internal Medicine C- Gastroenterology

Fig. 1 (A) Adjusted daytime electronic health record (EHR) usage according to specialty. (B) Adjusted after-hours EHR usage according to specialty. 


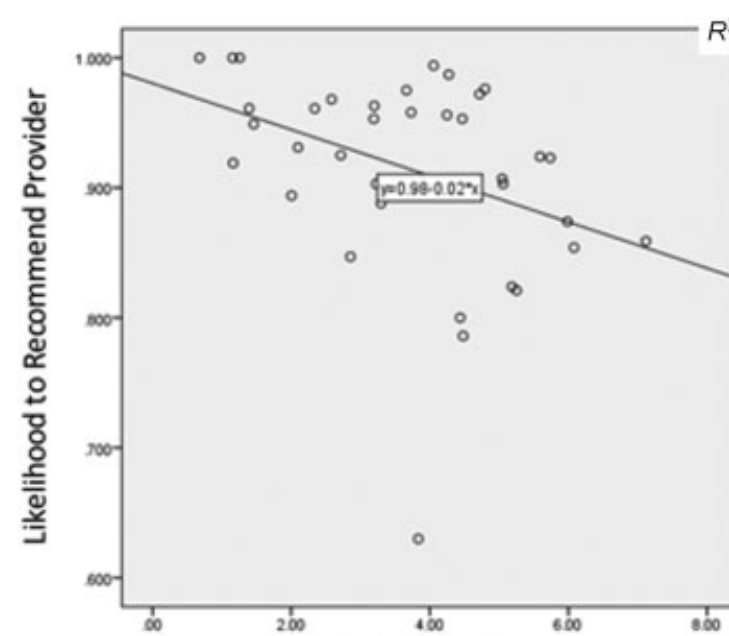

A Adjusted" Daytime Hours Logged into EHR

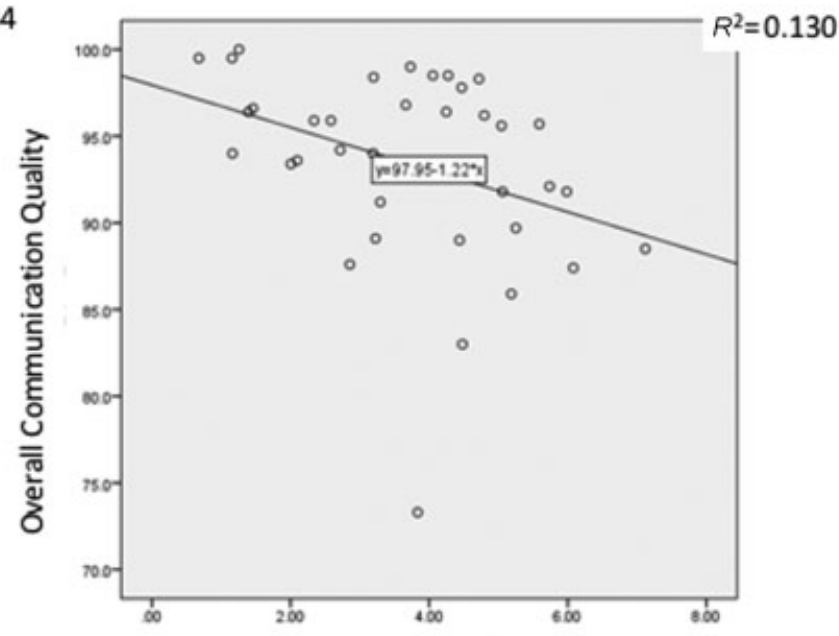

B Adjusted Daytime Hours Logged into EHR

Adjusted Daytime Hours: average monthly daytime hours provider logged into EHR divided by average monthly number of clinic days

Fig. 2 Adjusted daytime hours logged into electronic health record (EHR) and Clinician and Group Consumer Assessment of Healthcare Providers and Systems (CG CAHPS) performance.

Our work highlights the significant amount of time many physicians spend logged into the EHR, both during and after normal clinic hours. In addition, our study offers evidence to support the suspicions of many physicians: increased daytime EHR usage, potentially occurring in the exam room, may adversely impact patient-physician relationships. ${ }^{12}$ Physicians utilizing the EHR during the clinical encounter may appear distracted or disinterested to patients, thus impacting patient's perceptions of the physicians' communication skills and overall satisfaction with the care provided. Alternatively, high daytime EHR usage outside of the exam room may represent physician preparation for clinical encounters; in this scenario, physicians may believe they require a less thorough history from the patient. This practice could still negatively impact satisfaction scores; however, as prior research has demonstrated that many patients desire the opportunity to share information about their symptoms and issues during clinical encounters. ${ }^{13}$

Interestingly, we have demonstrated no association between increased after-hours EHR usage and patient satisfaction, suggesting that deferring documentation until after clinic may not result in improved patient-physician relationships or communication. This finding may be explained by the previous hypothesis: physicians who use the EHR to thoroughly prepare for patient visits (whether during daytime or after-hours) may be asking the patient fewer questions. Alternatively, heavy after-hours usage may either be a symptom or sequela of physician burnout. Previous research has demonstrated that physicians experiencing symptoms of burnout have less satisfied patients. ${ }^{14}$

Our pilot study is designed to be hypothesis-generating, and has several limitations which will need to be addressed in subsequent work. As we utilized the CG CAHPS survey, we had no information on individual patient characteristics which may inform patient satisfaction (e.g., sicker patients have been shown to be less satisfied with their care), ${ }^{15}$ or wait times of patients in clinic. Future work should incorporate additional details about patients to address possible confounders. Additionally, we were unable to obtain information on providers' specific practice patterns in regard to EHR usage in the exam room; knowing which providers use the computer in the exam room will help to better understand the relationship we have previously described between EHR usage and patient satisfaction. Lastly, future work should include a broader sample size to address possible differences in the relationship between EHR usage and patient satisfaction between medical specialties.

We believe this constellation of findings should encourage designers of EHRs think creatively about how to decrease the time burden of the EHR on clinicians. Changes such as increased interoperability between systems and streamlining workflows to minimize the number of clicks required to complete tasks ${ }^{16}$ may help to decrease the time burden of EHR use and potentially improve both the quality of life of our physician workforce and patient experience in the health care system.

\section{Multiple Choice Question}

Which of the following is true about the CG CAHPS Survey?

a. Elicits feedback from patients about their satisfaction with inpatient care

b. Is the only CMS-approved survey to assess patient satisfaction with health care experiences

c. Is developed by the Agency for Healthcare Research and Quality (AHRQ)

d. The overall communication quality domain is considered a surrogate for overall satisfaction with care

Correct Answer: The correct answer is c. The CAHPS family of surveys was developed by the AHRQ to assess patient satisfaction with various aspects of their health 
care experiences. The CG CAHPS is designed to assess patient satisfaction with ambulatory or outpatient care. Other surveys to assess patient satisfaction include: HCAHPS to assess satisfaction with inpatient care, Outpatient and Ambulatory Surgery (OAS CAHPS) to assess satisfaction with ambulatory surgery, and the CAHPS Hospice Survey to assess satisfaction with hospice care.

The CG CAHPS survey is quite robust, and measures patient satisfaction with several factors pertinent to their outpatient experiences including: getting timely appointments, helpful office staff, and how well providers communicate with patients. The survey item assessing the patient's likelihood of recommending the provider, however, is commonly considered a surrogate for overall satisfaction with care.

\section{Protection of Human and Animal Subjects}

This study was deemed exempt from Institutional Review Board review for quality improvement purposes.

\section{Conflict of Interest}

None.

\section{References}

1 Crossing the Quality Chasm. A New Health System for the 21st Century. Washington, DC: National Academy Press; 2001

2 Henry J, Pylypchuk Y, Searcy T, Patel V. Adoption of Electronic Health Record Systems among U.S. Non-Federal Acute Care Hospitals: 2008-2015. . ONC Data Brief 35; 2016

3 Gibbings-Isaac D, Iqbal M, Tahir MA, Kumarapeli P, de Lusignan S. The pattern of silent time in the clinical consultation: an observational multichannel video study. Fam Pract 2012;29(05):616-621

4 Kumarapeli P, de Lusignan S. Using the computer in the clinical consultation; setting the stage, reviewing, recording, and taking actions: multi-channel video study. J Am Med Inform Assoc 2013; 20(e1):e67-e75
5 Alkureishi MA, Lee WW, Lyons M, et al. Impact of electronic medical record use on the patient-doctor relationship and communication: a systematic review. J Gen Intern Med 2016;31(05): 548-560

6 Shanafelt TD, Boone S, Tan L, et al. Burnout and satisfaction with work-life balance among US physicians relative to the general US population. Arch Intern Med 2012;172(18):1377-1385

7 Peckham C. MedScape Lifestyle Report 2016: Bias and Burnout. 2016. Available at: https://www.medscape.com/slideshow/lifestyle-2016-overview-6007335. Accessed January 5, 2017

8 Shanafelt TD, Dyrbye LN, Sinsky C, et al. Relationship between clerical burden and characteristics of the electronic environment with physician burnout and professional satisfaction. Mayo Clin Proc 2016;91(07):836-848

9 Friedberg MW, Chen PG, Van Busum KR, et al. Factors affecting physician professional satisfaction and their implications for patient care, health systems, and health policy. Rand Health Q 2014;3 (04): 1

10 Dyer N, Sorra JS, Smith SA, Cleary PD, Hays RD. Psychometric properties of the Consumer Assessment of Healthcare Providers and Systems (CAHPS $\left.{ }^{\circledR}\right)$ Clinician and Group Adult Visit Survey. Med Care 2012;50(Suppl):S28-S34

11 Long C, Tsay EL, Jacobo SA, Popat R, Singh K, Chang RT. Factors associated with Patient Press Ganey Satisfaction Scores for ophthalmology patients. Ophthalmology 2016;123(02):242-247

12 Gadd CS, Penrod LE. Dichotomy between physicians' and patients' attitudes regarding EMR use during outpatient encounters. Proc AMIA Symp 2000:275-279

13 Simpson M, Buckman R, Stewart M, et al. Doctor-patient communication: the Toronto consensus statement. BMJ 1991;303 (6814):1385-1387

14 Haas JS, Cook EF, Puopolo AL, Burstin HR, Cleary PD, Brennan TA. Is the professional satisfaction of general internists associated with patient satisfaction? J Gen Intern Med 2000;15(02): $122-128$

15 Hall JA, Feldstein M, Fretwell MD, Rowe JW, Epstein AM. Older patients' health status and satisfaction with medical care in an HMO population. Med Care 1990;28(03):261-270

16 DiAngi YT, Longhurst CA, Payne TH. Taming the EHR (Electronic Health Record) - there is hope. J Fam Med 2016;3(06):1072 